\title{
Dynamics of Learning and Recall at Excitatory Recurrent Synapses and Cholinergic Modulation in Rat Hippocampal Region CA3
}

\author{
Michael E. Hasselmo, Eric Schnell, and Edi Barkai \\ Department of Psychology and Program in Neurosciences, Harvard University, Cambridge, Massachusetts 02138
}

\begin{abstract}
Hippocampal region CA3 contains strong recurrent excitation mediated by synapses of the longitudinal association fibers. These recurrent excitatory connections may play a dominant role in determining the information processing characteristics of this region. However, they result in feedback dynamics that may cause both runaway excitatory activity and runaway synaptic modification. Previous models of recurrent excitation have prevented unbounded activity using biologically unrealistic techniques. Here, the activation of feedback inhibition is shown to prevent unbounded activity, allowing stable activity states during recall and learning. In the model, cholinergic suppression of synaptic transmission at excitatory feedback synapses is shown to determine the extent to which activity depends upon new features of the afferent input versus components of previously stored representations. Experimental work in brain slice preparations of region $\mathrm{CA} 3$ demonstrates the cholinergic suppression of synaptic transmission in stratum radiatum, which contains synapses of the longitudinal association fibers.
\end{abstract}

[Key words: associative memory, presynaptic inhibition, medial septum, feedback attractor dynamics]

Hippocampal region CA3 contains extensive recurrent excitation, mediated by synapses of the longitudinal association fibers that arise from region CA3 pyramidal cells and terminate on other pyramidal cells along the septotemporal axis of the hippocampus (see Amaral and Witter, 1989, for review). Extensive recurrent excitation also appears in other cortical structures, such as the piriform (olfactory) cortcx (Haberly, 1985; IIaberly and Bower, 1989) and the neocortex, where the majority of excitatory synapses on pyramidal cells arise from other cortical pyramidal cells, rather than from thalamic afferents (Douglas and Martin, 1990). Thus, recurrent excitation may have a predominant role in determining the dynamics of activity in many cortical structures. However, this strong recurrent excitation has the potential to cause serious problems for maintaining bounded activity. Positive feedback can result in exponential increases in the activity of individual neurons, and can cause activity to

\footnotetext{
Received Oct. 20, 1994; revised Mar. 2, 1995; accepted Mar. 8, 1995

This work was supported by a pilot grant from the Massachusetts Alzheimer's Disease Research Center, Office of Naval Research Young Investigator Award N00014.93 I 0595, and NIMH Award R29 MH52732-01 to M.E.H. We thank Joshua Berke for assistance to E.S. and E.B. on physiological experiments, Carl Van Vreeskwijk, Don Anderson, and Todd Troyer for discussion, and John Holena and Lester Miller for programming support to M.E.H.

Correspondence should be addressed to Michael E. Hasselmo, Department of Psychology, Room 920, Harvard University, 33 Kirkland Street, Cambridge, MA 02138 .

Copyright (C) 1995 Society for Neuroscience $0270-6474 / 95 / 155249-14 \$ 05.00 / 0$
}

spread to a large percentage of neurons within a cortical region (Minai and Levy, 1994). In addition, the spread of activity across previously modified synapses during learning could result in excessive enhancement of synaptic strength within a cortical region (Hasselmo et al., 1992; Hasselmo, 1994a).

In models of the cortex with recurrent excitatory synapses, these problems have been avoided with unrealistic features. Models that use excitatory feedback to perform associative memory function commonly prevent runaway excitatory activity by limiting neuronal output with sigmoid input-output functions (Anderson, 1983; Hopfield, 1984; Amit, 1988). Maximum neuronal firing rate is, indeed, limited by the dynamics of voltagedependent channels, but this theoretical limitation is considerably higher than the maximal firing rate observed in awake behaving animals during recording from the hippocampus (Muller et al., 1987; Eichenbaun et al., 1988; Oto and Eichenbaum, 1992; Wilson and McNaughton, 1993) or neocortical structures (Hasselmo et al., 1989a,b). A large number of models have addressed the problem of low firing rates in networks performing associative memory function with attractor dynamics (Amit and Treves, 1989; Treves, 1990; Treves, 1991). The most biologically realistic solutions to this modeling problem incorporate shunting inhibition (Abbott, 1991, 1992). Shunting inhibition has also been utilized to limit network activity in models focused on sequences rather than fixed point attractors (Minai and Levy, 1994; Prepscius and Levy, 1994). Here, a novel network that maintains bounded, stable attractor states with feedback inhibition is analyzed.

In previous models of learning in networks with recurrent excitation, runaway synaptic modification was prevented by clamping the activity of the network to the input pattern during learning. Though this technique was used in a large number of models, no physiological mechanism was presented. Recently, it has been shown that the selective suppression of synaptic transmission at excitatory feedback synapses can provide such a mechanism (Hasselmo et al., 1992; Hasselmo, 1993, 1994; Hasselmo and Schnell, 1994). Selective suppression of excitatory recurrent synapses has been shown for substances that activate muscarinic cholinergic receptors (Hasselmo and Bower, 1992; Hasselmo et al., 1994a), GABA ${ }_{B}$ receptors (Ault and Nadler, 1982; Tang and Hasselmo, 1994) and noradrenergic receptors (Vanier and Bower, 1992; Scanziani et al., 1993). Here, it is shown that regulating the level of cholinergic modulation determines the extent to which storage of new afferent input patterns depends upon previously stored patterns. For low levels of cholinergic modulation, the network recalls previously stored patterns unaltered by the new input. For high levels of cholinergic modulation, the network learns the new pattern with no elements 

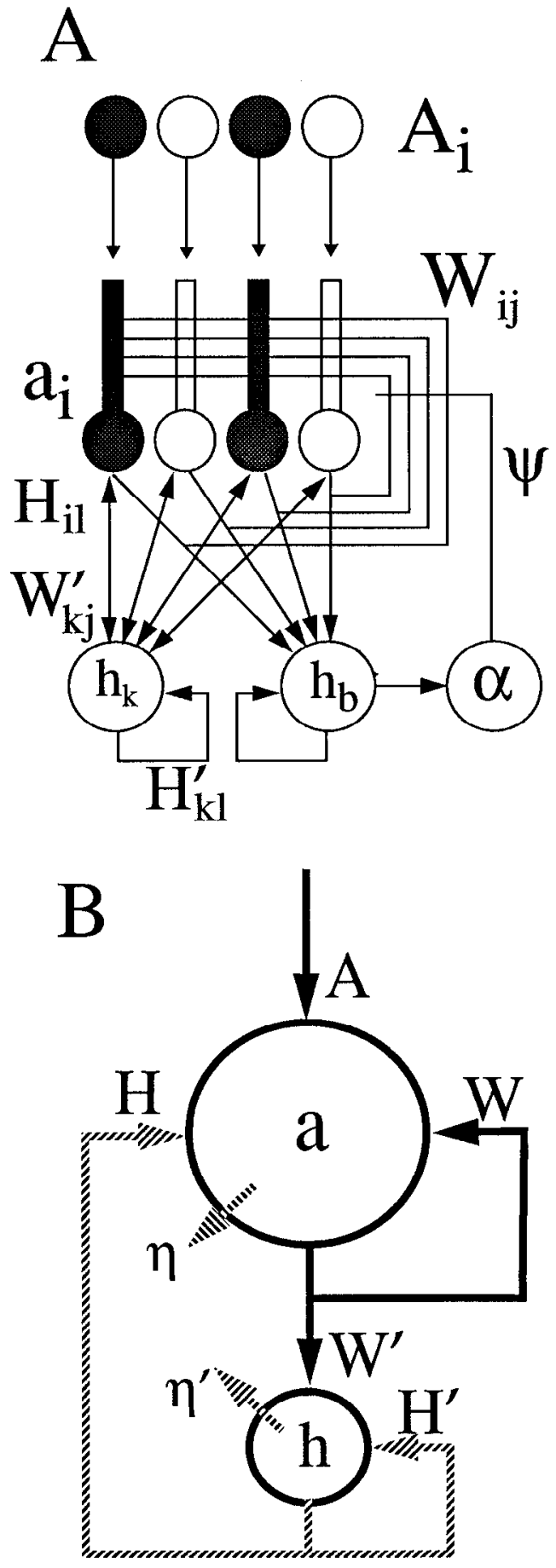

Figure 1. Schematic representation of the auto-associative network described here. A, Full network connectivity. Excitatory neurons with membrane potential $a_{i}$ receive afferent input $A_{i}$. Inhibitory interneurons with membrane potential $h_{k}$ receive afferent input $A^{\prime}{ }_{k}$ (not shown). Excitatory neurons contact each other via an excitatory fccdback matrix $W_{i j}$. Excitatory neurons contact inhibitory interneurons via connectivity matrix $W^{\prime}{ }_{k j}$ and receive inhibitory input via connectivity matrix $H_{i l}$. Inhibitory neurons receive inhibitory input via the connectivity matrix $H^{\prime}{ }_{k l}$. Feedback regulation of cholinergic modulation is mediated via excitatory connections to an inhibitory neuron with membrane potential $h_{b}$, which inhibits activity of the cholinergic neuron with membrane potential $\alpha$. Output from the cholinergic neuron $\psi$ influences a range of parameters in the network. $B$, For certain conditions, the dynamics of a population of neurons can be simplified as coupled equations with the membrane potentials of a single excitatory neuron $(a)$ and a single inhibitory neuron $(h)$ representing the activity of populations of excitatory and inhibitory neurons. Synaptic connectivity can be represented as an from previously stored patterns. For intermediate levels of cholinergic modulation, the network stores representations that chunk the new pattern together with previously stored patterns.

\section{Materials and Methods}

Computational modeling

Interaction of excitation and inhibition. Bounded self-sustained excitatory activity can be obtained in a network with the following equations for the membrane depolarization of excitatory pyramidal cells $(a)$ and the membrane depolarization of inhibitory interneurons $(h)$ :

$$
\begin{aligned}
& \Delta a_{i}=A_{i}-\eta a_{i}+\sum_{j} W_{i j}\left[a_{j}-\theta_{t l}\right]_{+}-\sum_{l} H_{i l}\left[h_{i}-\theta_{h}\right]_{+}, \\
& \Delta h_{k}=A_{k}^{\prime}-\eta^{\prime} h_{k}+\sum_{j} W_{k j}^{\prime}\left[a_{j}-\theta_{a}\right]_{+}-\sum_{l} H_{k l}^{\prime}\left[h_{l}-\theta_{h}\right]_{+},
\end{aligned}
$$

where $A$ represents the afferent input to a neuron, na represents the passive decay of membrane potential proportional to the difference from resting potential, $[a-\theta]_{+}$is a threshold linear output function of membrane potential, with zero output for values below $\theta$. W represents the matrix of cxcitatory synapscs arising from cortical pyramidal cells, and $H$ represents the matrix of inhibitory synapses arising from cortical inhibitory interneurons. For inhibitory interneurons, the membrane potential is represented by $h$. $W^{\prime}$ represents the matrix of excitatory synapses arising from cortical pyramidal cells and synapsing on inhibitory interneurons and $\mathrm{H}^{\prime}$ represents the matrix of inhibitory synapses between inhibitory neurons. The architecture of the full network is shown in Figure 1A. For mathematical analysis, the network was reduced to two neurons, one excitatory and one inhibitory, as shown in Figure $1 B$, allowing solution of the coupled pair of differential equations. Note that the simulations described here are fully connected, with synapses between all existing neurons. In addition, most simulations used a single feedback neuron to represent the population of neurons mediating feedback inhibition. These physiologically unrealistic features allow the model to function with smaller numbers of ncurons. Variations in the percentage connectivity of the network does not prevent attractor dynamics until connectivity reaches sufficiently low values (Van Vreeswijk and Hasselmo, unpublished data). Thus, the fully connected network here is used as an approximation to a much larger network with smaller percent connectivity.

The linear representation above does not take into account the reversal potentials of ionic currents. The equations can be modified in a simple manner to incorporate these reversal potentials, as follows:

$$
\begin{aligned}
\Delta a_{i}= & A_{i}-\eta a_{i}+\left(E_{\mathrm{Na}}-a_{i}\right) \sum_{j} W_{i j}\left[a_{j}-\theta_{a}\right]_{+} \\
& +\left(E_{\mathrm{Cl}}-a_{i}\right) \sum_{i} H_{i l}\left[h_{i}-\theta_{h}\right]_{+} \\
\Delta h_{k}= & A_{k}^{\prime}-\eta h_{k}+\left(E_{\mathrm{Na}}-h_{k}\right) \sum_{j} W_{k j}^{\prime}\left[a_{i}-\theta_{i}\right]_{+} \\
& +\left(E_{\mathrm{Cl}}-h_{k}\right) \sum_{l} H_{k l}^{\prime}\left[h_{i}-\theta_{h}\right]_{+}
\end{aligned}
$$

The linear form was used in the mathematical analysis and in the simulations shown in Figures 3 and 4 . The nonlinear form was used in the network simulations shown in Figures 5-7. Reversal potentials for membrane currents were expressed relative to the resting potential. Thus, $E_{\mathrm{Na}}=70, E_{\mathrm{Cl}}=0, E_{\mathrm{K}}=-10$. Threshold potentials were equivalent for all neurons: $\theta_{a}=\theta_{h}=8.0$. Afferent input was scaled to the magnitude of the decay constant allowing afferent input to depolarize the neurons to 10.0 (when $\eta=0.1, A=1.0$, when $\eta=0.01, A=0.1$ ). All passive decay parameters $\eta$ were set to the same value for an individual simulation $\left(\eta=\eta^{\prime}=\eta_{\| k}\right)$. Synaptic connectivity strengths varied in different simulations as described below.

Adaptation in excitatory neurons. The model included the phenomenon of adaptation observed in most cortical pyramidal cells (Barkai

$\leftarrow$

excitatory feedback synapse $W$, an excitatory input to the inhibitory interneuron $W^{\prime}$, an inhibitory input to the excitatory neuron $H$ and an inhibitory input to the inhibitory neuron $H^{\prime}$. The membrane potential of the excitatory and inhibitory neurons decays in proportion to the constants $\eta$ and $\eta^{\prime}$, respectively. The excitatory neuron is shown receiving afferent input $A$. 
and Hasselmo, 1994). This requires a simplified representation of intracellular calcium concentration in excitatory neurons. The adaptation characteristics of a single excitatory neuron were represented in the following highly simplified form:

$$
\begin{aligned}
& \Delta a_{i}=A_{i}-\eta a_{i}+\mu c_{i}\left(E_{\mathrm{K}}-a_{i}\right), \\
& \Delta c_{i}=\gamma\left[a_{i}-\theta_{c}\right]_{+}-\Omega c_{i},
\end{aligned}
$$

where $c$ is the intracellular calcium concentration, $\mu$ represents the strength of the calcium-dependent potassium current, $\gamma$ represents the strength of voltage-dependent calcium currents, $\Omega$ is the constant for diffusion of intracellular calcium, and $\theta_{c}$ is the threshold for activation of voltage-dependent calcium currents. Note that these equations describe just the intrinsic properties of an individual neuron, neglecting the terms for synaptic interactions. In simulations incorporating adaptation, the parameters were set to $\mu=0.01, \gamma=0.001, \Omega=0.001$, and $\theta_{c}=8.0$. This gave a reasonable simplified representation of the output characteristics of cortical pyramidal cells in response to intracellular current injection (Barkai and Hasselmo, 1994). The adaptation characteristics of a single neuron are shown in Figure 6.

Feedback regulation of cholinergic modulation. The model contained a representation of the level of acetylcholine $\psi$ within the entire cortical region, which varied between 0 and 1 . In addition, it incorporated several different effects of cholinergic modulation demonstrated experimentally. This included the selective suppression of excitatory intrinsic synaptic transmission $\left(1-\chi_{w} \psi\right)$, for which considerable experimental data exists (Hasselmo and Bower, 1992; Hasselmo and Schnell, 1994). In addition, it included the suppression of inhibitory synaptic transmission $\left(1-\chi_{H} \psi\right)$ (Pitler and Alger, 1992), the enhancement of excitatory synaptic modification (Burgard and Sarvey, 1990; Huerta and Lisman, 1993), the direct depolarization of inhibitory and excitatory neurons (Benardo and Prince, 1982; Madison and Nicoll, 1984; Barkai and Hasselmo, 1994), and the suppression of currents underlying adaptation (Barkai and Hasselmo, 1994; Madison and Nicoll, 1984). Simulation used the values $\chi_{W}=\chi_{H}=\chi_{H^{\prime}}=0.73, \chi_{W^{\prime}}=0.8, \chi_{\mathrm{AHP}}=1.0$ and depolarizing input $\chi_{\text {depul }}$ sufficient to bring resting potential to 4.0 . With inclusion of cholinergic effects, the activation equations took the following form.

$$
\begin{aligned}
\Delta a_{i}= & A_{i}-\eta a_{i}+\chi_{\text {depol }} \psi \\
& +\left(E_{\mathrm{Na}}-a_{i}\right) \sum_{j}\left(1-\chi_{W} \psi\right) W_{i j}\left[a_{i}-\theta_{a}\right]_{+} \\
& +\left(E_{\mathrm{Cl}}-a_{i}\right) \sum_{l}\left(1-\chi_{H} \psi\right) H_{i l}\left[h_{l}-\theta_{h}\right]_{+} \\
& +\left(E_{\mathrm{K}}-a_{i}\right)\left(1-\chi_{A H}\right) \mu c_{i} \\
\Delta h_{k}= & A_{k}^{\prime}-\eta^{\prime} h_{k}+\chi_{\mathrm{depol}} \psi \\
& +\left(E_{\mathrm{Na}}-h_{k}\right) \sum_{j}\left(1-\chi_{W} \psi\right) W_{k j}^{\prime}\left[a_{j}-\theta_{a}\right]_{+} \\
& +\left(E_{\mathrm{Cl}}-h_{k}\right) \sum_{l}\left(1-\chi_{H} \psi\right) H_{k j}^{\prime}\left[h_{j}-\theta_{h}\right]_{+} \\
\Delta c_{i}= & \gamma\left[a_{i}-\theta_{c}\right]_{+}-\Omega c_{i}
\end{aligned}
$$

Experimental evidence suggests that increased activity in cortical structures such as the hippocampus reduce the cholinergic modulation arising from basal forebrain structures such as the medial septum (McLennan and Miller, 1974). In the model, cholinergic modulation decreased due to the summed output from all neurons in the network, through the use of a feedback circuit in which single units represented the activation dynamics of basal forebrain populations of GABAergic neurons and cholinergic neurons. The GABAergic neurons had the same activation dynamics as the interneurons mediating feedback inhibition in the network, with the same parameters. The following activation dynamics applied for the cholinergic neuron output rate $\psi$ and membrane potential $\alpha$ :

$$
\psi=\Psi\left[\alpha-\theta_{\alpha}\right]_{+}, \Delta \alpha=A_{\psi}-\eta \alpha-H_{k}\left[h-\theta_{k}\right]_{+},
$$

where $\theta_{\alpha}$ is the output threshold for the cholinergic neuron, $A_{\psi}$ is tonic input to the cholinergic neuron present at all times during simulations to ensure continuous output in the absence of inhibition, and $H_{\downarrow}$ is the inhibitory synapse from GABAcrgic ncurons. Simulations used the values $\theta_{\alpha}=8.0$ and $\theta_{h}=8$. Other parameters varied as described below. The feedback regulation of cholinergic modulation is also summarized in Figure $1 \mathrm{~A}$.

Modification of excitatory synapses. Excitatory synapses between py

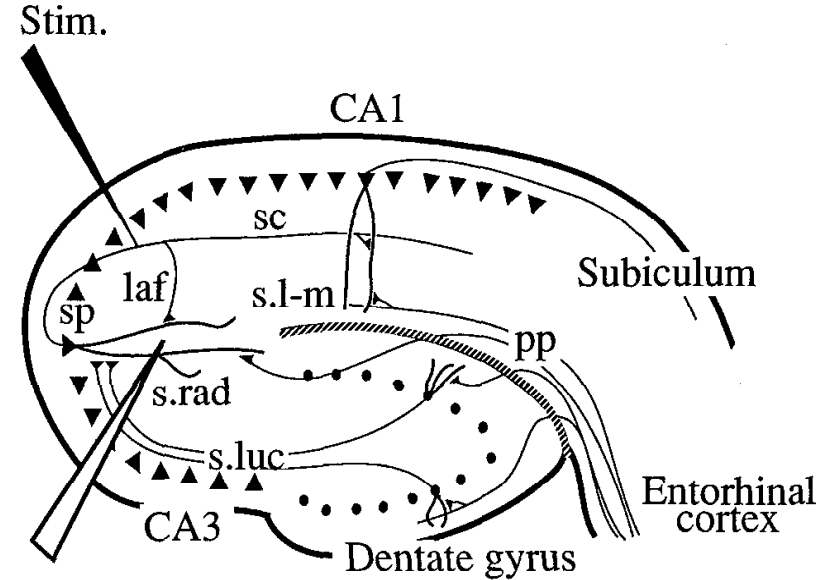

Rec.

Figure 2. Electrode placement in the brain slice preparation of the hippocampus for recording of synaptic potentials at excitatory recurrent synapses. Stimulating electrodes (Stim.) were placed in stratum radiatum near region $\mathrm{CA} 2$, to activate the longitudinal association fibers mediating recurrent excitation in hippocampal region CA3. Recording electrodes $(R e c$.) were placed in stratum radiatum of region CA3. laf, longitudinal association fibers; $s c$, schaffer collaterals; $s p$, stratum pyramidale; $s$. $l-m$, stratum lacunosum-moleculare; $p p$, perforant path; $s$. rad, stratum radiatum; s. luc, stratum lucidum.

ramidal cells in the model were modified continuously according to learning rules dependent upon postsynaptic activity $a_{i}$ and presynaptic activity $a_{i}$, in keeping with experimental evidence on the Hebbian nature of long-term potentiation (Kelso et al., 1986; Wigstrom et al., 1986). However, two versions were used, one of which depended upon the instantaneous levels of activity, the other that depended upon cumulative build-up of pre- and postsynaptic variables $s_{i}$ and $s_{i}$, which increased with separate dynamics. This could be construed as the buildup of pre- and postsynaptic calcium, or activation of pre- and postsynaptic second messengers such as protein kinase $\mathbf{C}$. Both rules also contained synaptic decay proportional to the current strength $W_{i j}$ and preor postsynaptic activity (scaled with the constants $\omega_{\text {prc }}$ and $\omega_{\text {post }}$ ), as a representation of long-term depression (Levy et al., 1990). Each rule had parameters for the overall modification rate $\kappa$ and the postsynaptic modification threshold $\theta_{w}$. The rate of synaptic modification was also scaled to the level of cholinergic modulation, as suggested by experiments showing cholinergic enhancement of long-term potentiation (Burgard and Sarvey, 1990; Huerta and Lisman, 1993). The cumulative learning rule took the form

$$
\begin{aligned}
\Delta W_{i j}= & \kappa\left(1-\chi_{w}[1-\psi]\right)\left(\left[s_{i}-\theta_{w}\right]_{+}-\omega_{\mathrm{pre}} W_{i j}\right) \\
& \times\left(\left[s_{j}-\theta_{w}\right]_{+}-\omega_{\mathrm{pos}} W_{i j}\right), \\
\Delta s_{i}= & \phi\left[a_{i}-\theta_{a}\right]_{+}-\beta s_{i}, \\
\Delta s_{j}= & \phi\left[a_{j}-\theta_{a}\right]_{+}-\beta s_{j} .
\end{aligned}
$$

The instantaneous learning rule took the form

$$
\begin{aligned}
\Delta W_{i j}= & \kappa\left(1-\chi_{w}[1-\psi]\right)\left(\left[a_{i}-\theta_{n}\right]_{+}-\omega_{\mathrm{pre}} W_{i j}\right) \\
& \times\left(\left[a_{j}-\theta_{w}\right]_{+}-\omega_{\text {post }} W_{i j}\right) .
\end{aligned}
$$

Connections to and from inhibitory interneurons were not modified in these simulations. In most simulations, weights were clipped at specific values to maintain them within the region of stable attractor dynamics. In this case, runaway synaptic modification applies not to the exponential enhancement of a single connection, but to the enhancement of additional undesired synapses. This clipping allowed stable learning for a broader range of parameters, but may not be physiologically realistic. Additional simulations demonstrated that stable synaptic modification in response to new patterns conld be obtained with synaptic enhancement limited only by decay proportional to synaptic strength. However, this required the use of much larger decay constants, which resulted in a loss of completion in response to degraded patterns, since the decay would delete additional synapses before the spread of activity could sufficiently activate neurons not receiving direct afferent input. This suggests that memor y function in real physiological networks may require limits on synaptic modification that do not involve decay of this 


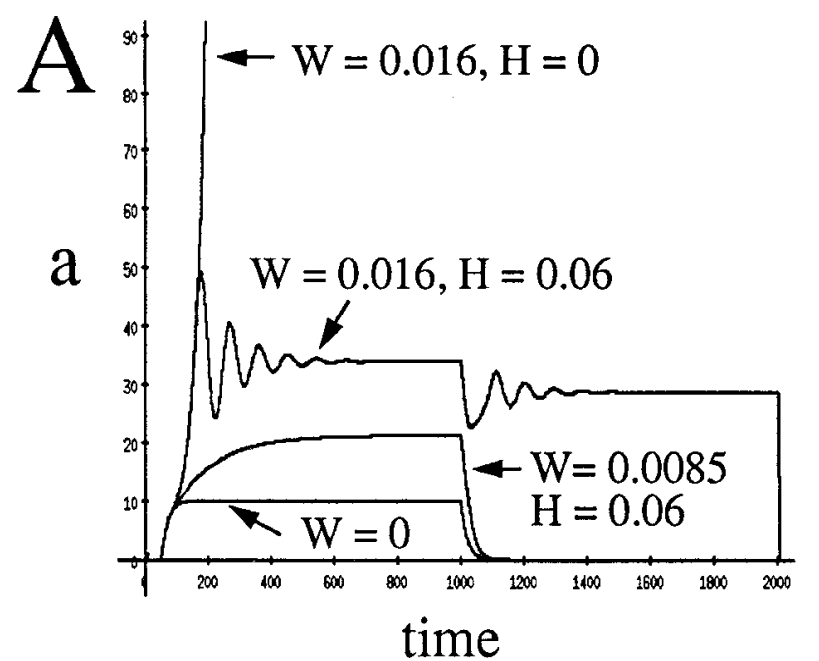

B
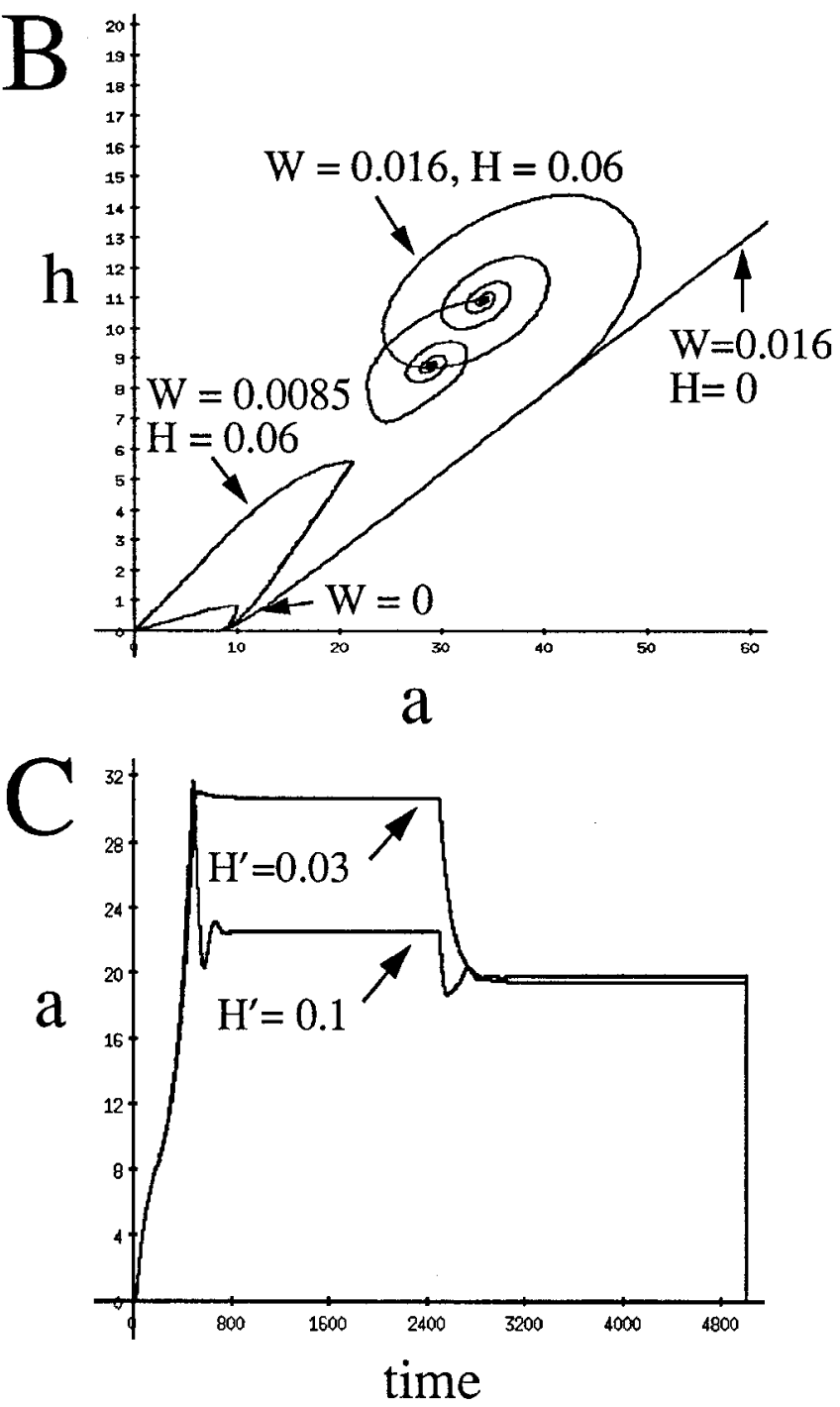

Figure 3. Capability for bounded, self-sustained activity in the network from Equation 1. Identical traces were obtained for a neuron in a network with multiple excitatory neurons, and for a single excitatory neuron coupled with one inhibitory neuron with parameters scaled to match the network. A, The membrane potential $(a)$ of an excitatory neuron plotted against time $(t)$ before, during, and after a square pulse of afferent input (from $t=50$ to 1000). $B$, For the same traces as $A$, the membrane potential $(a)$ of the excitatory neuron is plotted against the membrane potential $(h)$ of an inhibitory neuron to yield a phase type. Another possibility is that real physiological networks may contain considerably longer delays in the implementation of synaptic modification relative to the spread of activity. This would allow sufficient time for activation dynamics to approach a stable state before synaptic modification influences these dynamics.

\section{Brain slice physiology}

Cholinergic modulation was studied in brain slice preparations of hippocampal region $\mathrm{CA} 3$ to determine the whether suppression appears in this region similar to the suppression in region CA1. Cholinergic suppression of synaptic transmission was tested in stratum radiatum of region $\mathrm{CA} 3$ at the synapses of the excitatory recurrent longitudinal association fibers.

Using previously presented techniques (Hasselmo and Schnell, 1994), slices of the hippocampus were prepared from 21 albino Sprague-Dawley rats. Brains were removed from rats anesthetized with Halothane and rapidly immersed in chilled modified Ringer's solution with the following components: $\mathrm{NaHCO}_{3} 26, \mathrm{NaCl} 124, \mathrm{KCl} 2.5, \mathrm{KH}_{2} \mathrm{PO}_{4} 1.2$, $\mathrm{CaCl}_{2} 2.4, \mathrm{MgSO}_{4} 1.3$, and glucose $10 \mathrm{~mm}$. This same solution was used for storage of slices at least $1 \mathrm{hr}$ prior to recording in vials bubbled with $95 \% \mathrm{O}_{2} / 5 \% \mathrm{CO}_{2}$.

For recording from region $\mathrm{CA} 3$, slices were mounted on nylon mesh in a submersion-type slice chamber perfused with Ringer's solution (36 $\pm 1.0^{\circ} \mathrm{C}$ ) at $4 \mathrm{ml} / \mathrm{min}$. Slices were transilluminated to allow visually guided placement of recording electrodes in stratum radiatum of region CA3. See Figure 2 for summary of electrode placement. For activation of synaptic potentials, stimulating electrodes were placed in stratum radiatum near region $\mathrm{CA} 2$, except in the case of synaptic potentials recorded before, during, and after perfusion with $20 \mu \mathrm{M}$ carbachol, which were activated with stimulating electrodes placed between the dentate gyrus and the recording electrode. Bipolar stimulating electrodes consisted of twisted strands of teflon-insulated 0.002" diameter platinum-iridium wire (A-M Systems, Inc.).

Stimulation pulses of $0.1 \mathrm{msec}$ duration were delivered at $5 \mathrm{sec}$ intervals using a Neurodata PG4000 stimulator with stimulus isolation units. Evoked potentials were amplified using a custom built extracellular amplifier and recorded using custom written software on a Gateway $2000386 \mathrm{SX}$ computer. After synaptic potentials were initially obtained, they were observed regularly to determine stability. Experimental procedures were commenced when the amplitude of the EPSP did not change for a $10 \mathrm{~min}$ period. Perfusion protocols were then commenced. Averages of 10 successive digitized traces in a given layer were obtained before, during, and after perfusion of the cholinergic agonist carbamylcholine chloride (Carbachol), obtained from Sigma Chemicals (St. Louis, MO).

Effects of cholinergic modulation on synaptic potentials in stratum

$\leftarrow$

plane representation. Parameters took the values $W^{\prime}=0.0042, H^{\prime}=0$, $\eta=\eta^{\prime}=0.01$. Different values of $W$ and $H$ determined the behavior of the network as listed below: $W=0$ : with no feedback excitation, the neuron shows only passive membrane properties, charging up asymptotically to $A / \eta$ during afferent input, then decaying passively to zero after input is terminated. $W=0.016, H=0$ : with feedback excitation in the absence of feedback inhibition, presentation of suprathreshold afferent input causes the membrane potential to grow rapidly to large values. This will occur for: $W>\eta+H W^{\prime} /\left(H^{\prime}+\eta^{\prime}\right)$ or $W>$ $\eta+\eta^{\prime}+H^{\prime} . W=0.0085, H=0.06$ : feedback inhibition keeps activity bounded, but insufficient excitatory feedback $(W<\eta)$ prevents sustained activity. The membrane potential decays to zero after afferent input ceases. $W=0.016, H=0.06$ : for certain parameters, the network reaches a stable, self-sustained activity level. During afferent input, the activity rises to a stable equilibrium. After afferent input ceases, the equilibrium changes slightly, but the network displays persistent suprathreshold activity. These parameters satisfy $\eta<W<\eta+H W^{\prime} /\left(H^{\prime}+\right.$ $\eta^{\prime}$ ) and $W<\eta+\eta^{\prime}+H^{\prime}$. For the simulation, self-sustained activity appears in a more restricted range of parameters, because the stability criteria described mathematically apply to a network starting with initial conditions sufficiently near the equilibrium point that activity does not fall below threshold. $C$, Addition of inhibitory feedback to the inhibitory neurons makes for a more rapid approach to the equilibrium state at low membrane potential values. The membrane potential dynamics are shown for the parameters $W=0.016, W^{\prime}=0.0042$, and $H=0.06$, with $H^{\prime}=0.03$ or 0.1 . 

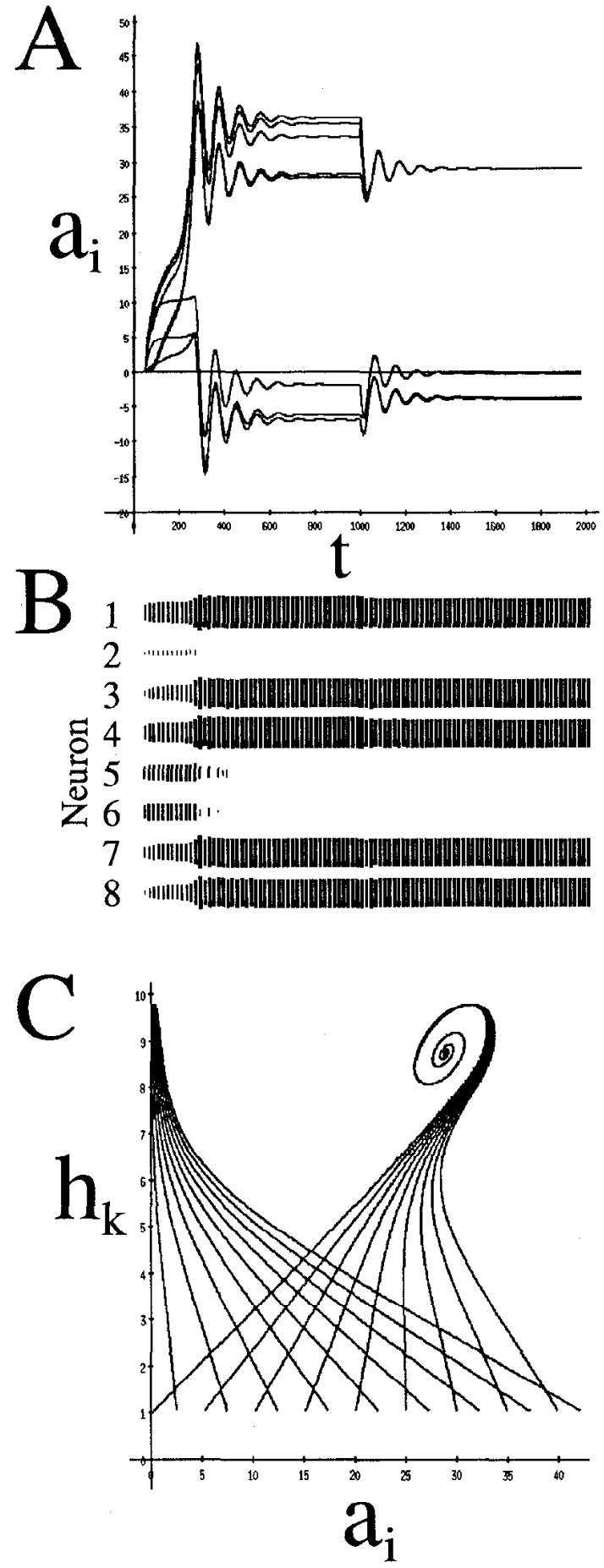

Figure 4. Dynamics of recall in a network of neurons. A, The membrane potentials $a_{i}$ of eight excitatory neurons plotted against time before, during, and after presentation of different amplitudes of afferent input to five neurons (from $t=50$ to 1000). The neurons are connected by nonuniform excitatory connections, with five neurons strongly interconnected. Note that two neurons that do not receive afferent input increase to high activity levels, while two neurons receiving afferent input are inhibited to levels below zero. This demonstrates that recall dynamics driven by feedback inhibition and excitation can dominate over the features of afferent input. Upon removal of afferent input, the five active neurons settle to uniform membrane potentials despite nonuniform excitatory connectivity. $B$, Plot of the same data shown in $A$ with size of black squares representing amplitude of membrane potential. $C$, Phase plane plot of the membrane potential of 18 excitatory neurons plotted against a single inhibitory neuron. Nine neurons are connected by uniform excitatory connections, while the other nine are radiatum were tested with perfusion of carbachol at $1 \mu \mathrm{M}, 5 \mu \mathrm{M}, 20$ $\mu \mathrm{M}$, and $100 \mu \mathrm{M}$. Once carbachol reached its maximal effect, washout was commenced with normal perfusant. Carbachol was considered to have reached its peak effect when the (altered) size of the EPSP remained stable for at least $3 \mathrm{~min}$ following the onset of drug perfusion, or no effect was observed for $10 \mathrm{~min}$. A slice was never tested at the same drug concentration more than once. EPSPs were verified using a solution with low calcium ( $\left.200 \mu \mathrm{M} \mathrm{CaCl}_{2}, 8 \mathrm{~mm} \mathrm{MgSO}_{4}\right)$.

Synaptic potentials were digitized for measurement of the exact value for the peak negative deflections of both the initial slope and the EPSP of a given trace by manual placement of a cursor. The slope calculation was performed manually on that portion of the synaptic potential for which the slope was essentially linear. The effect of carbachol was calculated for the percent change in both amplitude and slope of EPSPs.

\section{Results}

Stable attractor states with recurrent excitation

The interaction of pyramidal cells and inhibitory interneurons allows the network to show stable attractor states, as shown in Figure 3. For certain parameters, afferent input causes the network to enter a nonzero attractor state, showing neither unbounded exponential increases, nor decay back to zero activity. In addition, when afferent input was removed, this network was capable of maintaining a stable nonzero attractor state, avoiding decay back to zero activity. Thus, without using an explicit maximum activity level, a simple network with feedback inhibition can enter stable activity states.

Analysis of the equations allows estimation of conditions in which activity will enter a stable nonzero attractor state. We can approximate the network of neurons with distributed excitatory and inhibitory connections shown in Equation 1 and Figure $1 A$ as a pair of neurons, one excitatory and one inhibitory, with the connectivity shown in Figure $1 B$ (see appendix). For example, when the values of all the parameters are homogeneous (e.g., the strength of individual synaptic connections are all the same), then the network of neurons in Equation 1 can be represented as a single excitatory and inhibitory neuron with excitatory connections with synaptic strength $W=N^{*} W_{i j}$ and $W^{\prime}=N^{*} W_{i j}^{\prime}$. The traces shown in Figure 3 are equivalent for a single neuron with excitatory feedback and a population of neurons with excitatory and inhibitory connections scaled in this manner. The solution to the coupled equations with excitatory feedback $W$ is shown in the appendix.

Although this analysis represents a simplification, the dynamics of a network with more broadly distributed synaptic weights, intrinsic parameters, and initial conditions consistently tend toward the mean dynamics expressed by these equations, as shown in Figure 4. With heterogeneity in various parameters such as afferent input and excitatory connectivity, the activity of individual neurons can still be described in terms of these coupled equations, with different scaling of the constants. With a range of values of afferent input or initial conditions, the network tends toward stable homogeneous attractor values, particularly after afferent input has ceased.

The equilibrium state of the network can be obtained by setting $d a=d h=0$. This allows computation of the excitatory neuron equilibrium state $a=Q$, shown in the appendix. Note that for activity to persist after removal of the afferent input, the

$\leftarrow$

connected by much weaker excitatory connections. The initial conditions for each neuron range from 0 to 45 , but the dynamics result in the nine neurons with weak connections decaying to zero, and the nine neurons with stronger excitatory interconnectivity approaching a stable equilibrium state at about 30 . 
equilibrium state $Q$ must be larger than the output threshold of the pyramidal cell equation $\theta_{a}$. Otherwise, as the network approaches its equilibrium state, it falls below threshold and the activity decays to zero.

Ability to hold a stable recall state without afferent input. As shown in the appendix, computation of the eigenvalues of Equation 1 yields a notion of how the stability of nonzero activity states depends upon the relative magnitude of excitatory synaptic feedback $W$ and other synaptic and intrinsic parameters in the linear portions where $a>\theta_{a}$ and $h>\theta_{h}$. In particular, this allows analysis of the range of parameters within which the network can maintain stable excitatory activity without afferent input $A$. The parameters used here are the synaptic connectivity parameters shown in Figure $1 B$. The different conditions described below correspond to different traces in Figure 3.

(1) For $W<\eta$. Excitatory feedback does not overcome the passive decay of the membrane potential. Without afferent input $A$, the network will decay back to an equilibrium state at resting membrane potential $(a=0)$. For example, $W=0$ and $W=$ 0.0085 in Figure $3 A$.

(2) For $W>\eta+H W^{\prime} /\left(\mathrm{H}^{\prime}+\eta^{\prime}\right)$ or $W>\eta+\eta^{\prime}+H^{\prime}$. At least one of the eigenvalues is greater than zero, and the activity of the network will move away from the equilibrium point $Q$, showing exponential increases, or oscillations of increasing amplitude. This increased activity may correspond to the development of epileptic seizure activity in cortical networks. For examplc, the trace for $W=0.016, H=0$ shows runaway excitatory activity in Figure $3 A$.

(3) For $\eta<W<\eta+H W^{\prime} /\left(H^{\prime}+\eta^{\prime}\right)$ and $W<\eta+\eta^{\prime}+$ $H^{\prime}$. Both eigenvalues are less than zero, and the system remains bounded. At the same time, excitatory feedback gives the network the capacity to hold a particular recall state in the absence of afferent input $A$. Given these conditions, the values of different parameters determine whether the system will approach the equilibrium point with exponential decay, or with oscillations. If $\left[(W-\eta)+\left(-H^{\prime}-\eta^{\prime}\right)\right]^{2}<4\left((W-\eta)\left(-H^{\prime}-\eta^{\prime}\right)+W^{\prime} H\right)$, the network will approach a stable activity state with a damped oscillation, such as those seen in Fig. $3 A$ and $B$ for the trace with the parameters $W=0.016$ and $H=0.06$. (Other parameters in this figure were $W^{\prime}=0.0042$ and $H^{\prime}=0, \eta=0.01, \eta^{\prime}=$ 0.01, $A=0.1, \theta_{a}=8.0, \theta_{h}=8.0$.) The damped oscillations apparent in the figure do not appear for higher values of the equilibrium state, but simulations are shown for lower values because of the low firing rates of most cortical neurons. As shown in Figure $3 C$, adding inhibitory feedback to inhibitory interneurons $\left(H^{\prime}=0.03\right)$ causes a more rapid approach to equilibrium state, though higher values $\left(H^{\prime}=0.1\right)$ reintroduce damped oscillations as the equilibrium point decreases in amplitude. The approach is also more rapid for the nonlinear version of the equations, as shown in Figures 5 and 7.

The equilibrium state held by the network in the absence of afferent input can be detcrmined from the equilibrium equation $Q$, with $A=A^{\prime}=0$. This equilibrium state can be the same as the equilibrium state with afferent input if feedforward activation of inhibition $\left(A^{\prime}\right)$ satisfies the following requirement:

$$
A^{\prime}=\frac{A\left(\eta^{\prime}+H^{\prime}\right)}{H}
$$

For the network to hold this equilibrium state, the equilibrium state $Q$ must be higher than the output threshold. Solving for $Q$ $>\theta_{a}$, demonstrates that this requires the following:

$$
\eta \theta_{a}<H \theta_{h}-\frac{H H^{\prime} \theta_{h}}{\eta^{\prime}+H^{\prime}}
$$

The capacity to hold a particular activity state without external input may reflect an important processing characteristic of cortical structures, which here depends upon inhibition to maintain stability.

\section{Dynamics of learning and recall in the network}

As described in previous publications, associative memory function with recurrent excitation requires different dynamics during learning than during recall (Hasselmo et al., 1992; Hasselmo, 1993, 1994; Hasselmo and Bower, 1993). Here, a mechanism is proposed that allows internal self-regulation of the dynamics of learning and recall. This mechanism uses the summed output of the modeled cortical pyramidal cells to decrease the cholinergic modulation of the region, as described in Materials and Methods.

Equilibrium during learning and recall. In this framework for feedback control, cholinergic modulation is strong when there is no activity within the network, resulting in tonic suppression of intrinsic excitatory synapses. When a new afferent input pattern is first presented to the network, the input does not match the pattern of intrinsic connectivity. Activity of individual neurons may cross threshold due to afferent input, but recurrent excitation does not drive the activity to higher values. This means that cholinergic modulation remains strong. For example, in the simplified scheme presented above, imagine that the excitatory feedback starts with a weak connectivity $W(0)$, and in addition is suppressed by cholinergic modulation $\left(1-c_{W}\right)$. This results in an initial equilibrium, which is smaller than the threshold for decreasing cholinergic modulation $(v)$. If $W(0)$ is smaller than $\eta$, the excitatory feedback does not drive the network to an equilibrium, which will persist without afferent input. However, as the intrinsic feedback connectivity $W$ increases, the value of the equilibrium increases, eventually surpassing the threshold for feedback regulation of cholinergic modulation. As this threshold is passed, the suppression of excitatory feedback is discontinued, and the equilibrium potential increases further. Now the activity in the network is dominated by excitatory feedback, and the network has made the transition from learning to recall.

Presentation of a familiar pattern to the network results in a level of activity that immediately surpasses the threshold for fccdback regulation of cholincrgic modulation. In this casc, cholinergic modulation is immediately suppressed, before any synaptic modification takes place. The network immediately goes to a recall equilibrium dominated by the excitatory recurrent synapses.

Even without cholinergic modulation, the network with recurrent excitation described here is already more resistant to runaway synaptic modification than the simpler networks described in previous articles (Hasselmo et al., 1992, 1993, 1994). This is because the absence of attractor dynamics in those previous networks allowed partial activity from several different patterns to persist in the neurons. In contrast, the feedback inhibition in the network described here can eliminate most diffuse activity, preventing undesired neuronal activity and, hence, preventing undesired synaptic enhancement. However, cholinergic modulation provides a broader variety of dynamical characteristics within the network, as described in the next section.

Cholinergic levels determine state of pure learning, chunking, or pure recall. The network can show different patterns of response to afferent input, dependent upon the level of tonic ac- 

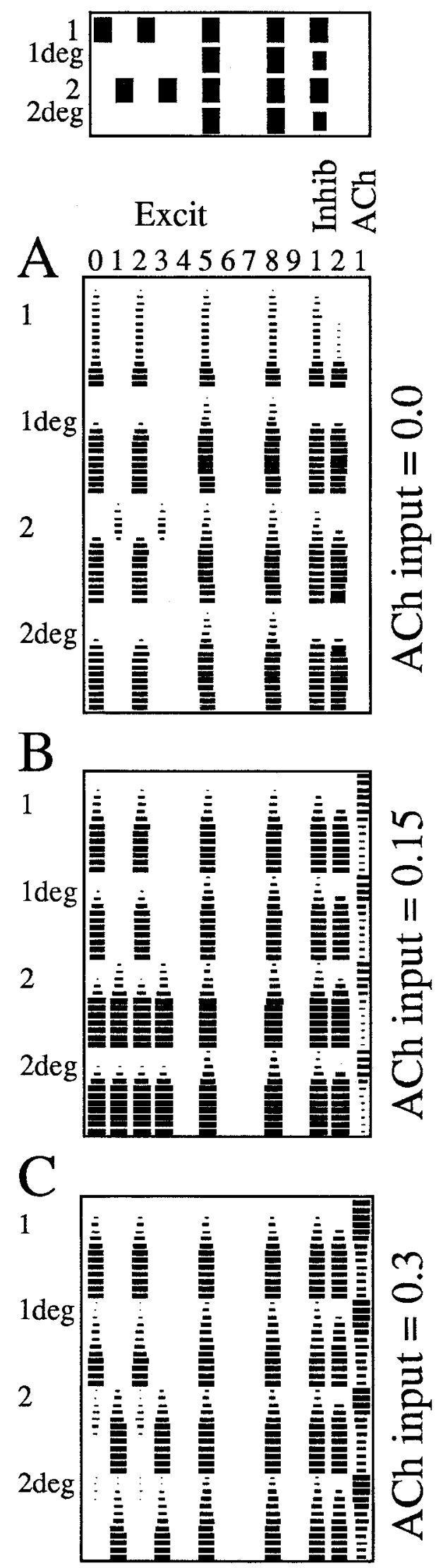

Figure 5. Self-regulated learning and recall of two highly overlapping patterns in the auto-associative network. Afferent input patterns are shown at top, with black squares representing active input lines. Pattern $I$ and 2 each contain four active input lines, with two lines in common between the patterns. Degraded versions of the input patterns are lack tivation of cholinergic innervation. With no afferent input to the cholinergic neuron, cholinergic modulation is absent. In this case, the network can learn the initial presentation of a novel pattern, but any subsequent presentation of a similar pattern results in recall of the initially learned pattern, as shown in Figure $5 A$. The representation of the pattern is not modified, but this previously stored representation dominates recall dynamics. Only presentation of orthogonal patterns will allow storage of a completely new representation. This could correspond to behavioral situations in which no learning is deemed necessary, and all behavior is guided by how much sensory stimuli resemble previously learned stimuli.

In contrast, with somewhat stronger input to the cholinergic neuron $(A \psi=0.15)$, the network shows different dynamics, as shown in Figure $5 B$. In this case, recall of the previously learned pattern is partially suppressed, allowing components of the new overlapping pattern to modify the representation. Components of both patterns become simultaneously active, and associations are formed between every element of each pattern. In previous articles (Hasselmo et al., 1992; Hasselmo, 1993, 1994; Hasselmo and Bower, 1993), this has been described as interference during learning and avoided as a problem, but in some cases, this combination of elements of different patterns can be useful to enhance the learning of new information. This phenomenon could be interpreted as chunking behavior. The ability to control whether chunking would be utilized may be an important role of cholincrgic modulation.

Finally, if input to the cholinergic neuron is increased further, recall is completely suppressed, and the network can separately learn even patterns which strongly overlap with previous patterns, as shown in Figure $5 \mathrm{C}$. Here, input to the cholinergic neuron is set to $A \psi=0.3$. In this case, after learning of the first pattern, a degraded version of the first pattern can still recall the first paltern, but presentation of a second strongly overlapping pattern does not have sufficient overlap to cause recall of the first pattern. Instead, the second pattern is learned as a novel representation. In this case, strong cholinergic modulation completely prevents recall of previously learned patterns from interfering with the learning of new patterns. The level of input to

\footnotetext{
$\leftarrow$

ing two active input lines. $A-C$, Pattern of activity in the network during sequential presentation of pattern 1 , degraded pattern 1, pattern 2, and degraded pattern 2 . The activity of each of 10 excitatory neurons $(E x-$ cit), two inhibitory neurons (Inhib), and one cholinergic neuron (ACh) is shown for every 50th simulation step, with size of black squares representing activity level. $A$, With no cholinergic modulation activity tends toward previously stored patterns (input to $\mathrm{ACh}$ neuron $=0.0$ ). During presentation of pattern 1, the network shows lower activity until synaptic calcium crosses threshold, strengthening excitatory feedback, and causing an increase in activity. Subsequent presentation of a degraded version of pattern 1 rapidly evokes the full stored pattern. Subsequent presentation of overlapping pattern 2 initially evokes a different pattern of activity, but eventually the network settles to the previously stored pattern $1 . B$, With moderate cholinergic modulation, overlapping patterns are chunked together. Learning of pattern 1 is followed by presentation of pattern 2 . In this case, feedback regulation of cholinergic modulation allows both activity patterns to be evoked, causing a combined representation of both patterns to be stored, such that the degraded version of pattern 2 evokes elements of both pattern 1 and 2. C, With strong cholinergic modulation, new overlapping patterns can be stored without interference from previous patterns. After learning of pattern 1, presentation of pattern 2 initially evokes a small portion of pattern 1 , but the suppression of synaptic transmission prevents this from dominating, and the network now stores pattern 2. Subsequent presentation of the degraded version of pattern 2 evokes only pattern 2 .
} 
the cholinergic neuron has a similar role to that of the vigilance parameter in adaptive resonance theory, though the structure of the network differs radically (Carpenter and Grossberg, 1993). The parameters used in the simulations shown in Figure 5 were as follows: $\eta=0.01$ (all neurons), $A=0.1$, starting $W=$ 0.000002 , maximum $W=0.00055, W^{\prime}=0.0008, H=-0.0035$, $H^{\prime}=-0.0055, W^{\prime}{ }_{b}=0.001, H^{\prime}{ }_{b}=-0.0055, H_{\psi}=-0.0008$, $\Psi=0.1$. The cumulative version of the learning rule was used with the parameters: $\phi=0.5, \beta=0.001, \omega_{\text {pre }}=0.002, \omega_{\text {post }}=$ $0.00002, \kappa=0.5, \theta_{w}=0.05$. Note that the adaptation parameter $\mu$ was set to zero for this simulation, preventing any adaptation.

Adaptation allows transitions between stable recall states. The interaction of feedback excitation and inhibition alone can cause the network to enter a stable attractor state, but cannot remove the network from that state. In single-unit recording, neurons of CA3 do not show long-term persistent activity in the absence of afferent input. Thus, once an attractor state has been approached, it must be terminated. This is where slower processes mediated by potassium currents might play a role in network dynamics. The activation of the calcium-dependent potassium current, or of $\mathrm{GABA}_{\mathrm{B}}$ potassium currents may play the role of pushing the network out of stable attractor states, allowing presentation of additional input to determine the response of the network.

Figure 6 shows the approximation of neuronal adaptation characteristics using the simplified representation presented in Equation 3. Incorporation of a variable representing intracellular calcium concentration and effects of this variable on calciumdependent potassium currents provides a simple model of both the adaptation of firing rate, and the afterhyperpolarization present after suprathreshold current injection ceases. The simulation shown in Figure 6 used parameters listed in the methods section for the characteristics of adaptation. There were no synaptic connections in this simulation, and $\eta-0.1$.

Incorporation of these effects in the simulation allow termination of the attractor states, as shown in Figure 7. Each new input pattern is learned, and after removal of afferent input, the activity persists for a period of time. However, eventually the adaptation currents decrease activity to below the output threshold and the network becomes inactive. Subsequent presentation of another pattern allows learning or recall without interference from the previous stable activity state. During recall, degraded patterns set different initial conditions, but after removal of afferent input, activity approaches the same stable states approached during learning before adaptation terminates activity. The simulation shown in Figure 7 . used the following parameters: $\eta=0.1$ (all neurons), $\mathrm{A}=1$, starting $W=0.000002$, maximum $W=0.003, W^{\prime}=0.005, H=-0.015, H^{\prime}=-0.025$, $W^{\prime}{ }_{\mathrm{b}}=0.005, H^{\prime}{ }_{b}=-0.025, A_{\psi}=0.9, H_{\psi}=-0.004, \Psi=1.0$. The instantaneous learning rule was used with the parameters: $\omega_{\text {pre }}=0, \omega_{\text {post }}=0, \kappa=0.5, \theta_{w}=8.0$. The adaptation parameters took the values listed in Materials and methods.

\section{Cholinergic suppression of synaptic transmission in region CA3}

Computational modeling demonstrates that cholinergic suppression of synaptic transmission can determine whether afferent input that overlaps with previously learned patterns will evoke just the previously stored pattern, will cause chunking of new information with previously learned information, or will result in learning of the afferent input as novel, with no component of the previously learned pattern. This requires cholinergic sup-
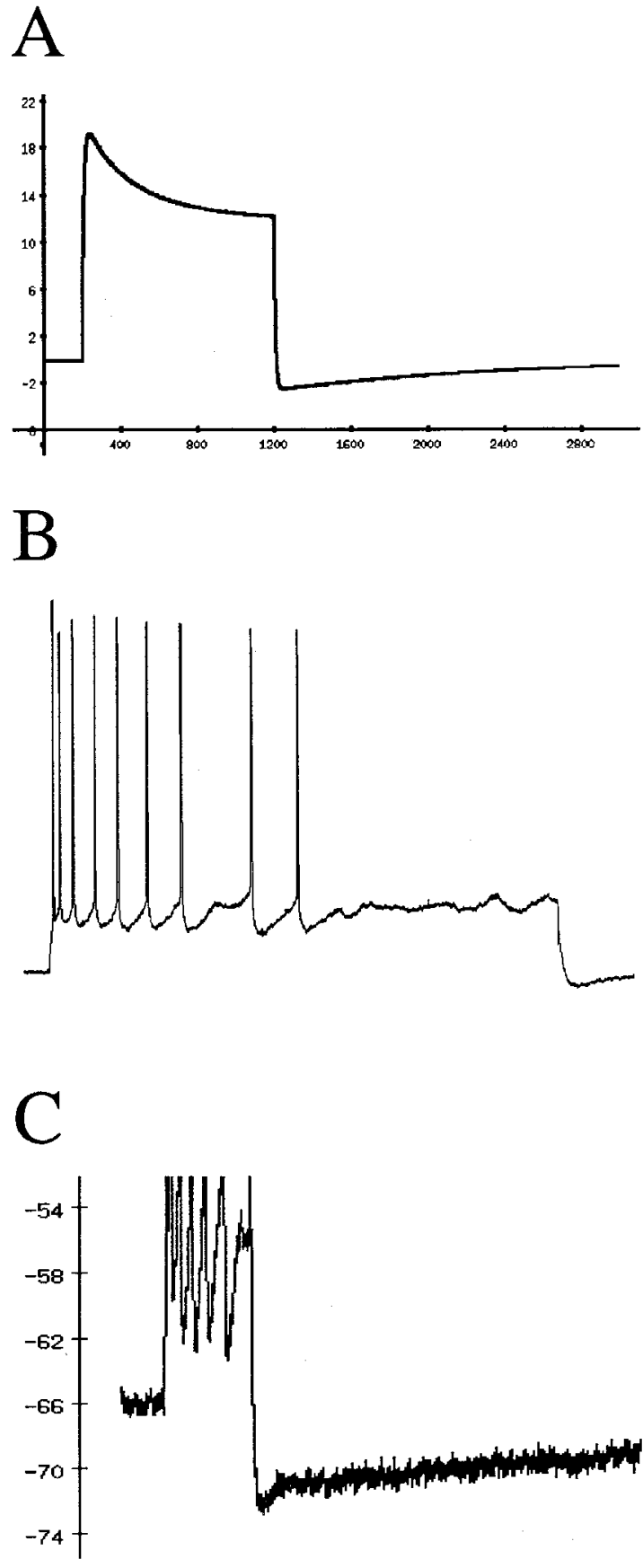

Figure 6. A, Adaptation characteristics of a single simulated neuron, with the dynamics shown in Equation 3. The membrane potential of the neuron is plotted against time before, during, and after a simulated current injection (from 50 to $1000 \mathrm{msec}$ ). The membrane potential shows an initial sharp increase followed by an exponential decay due to increased intracellular calcium concentration and activation of calcium-dependent potassium currents. The rate of adaptation shown here corresponds to a typical pattern of adaptation found in recordings from a population of piriform cortex pyramidal cells (see $B$ ). Following the end of the simulated current injection, the membrane potential goes to values below zero, due to persistent activation of potassium currents. This corresponds to the slow afterhyperpolarization found in cortical pyramidal cells (see $C$ ). $B$, Adaptation in a real cortical pyramidal cell. The initial high firing rate of the neuron drops off in a manner similar to the decrease in suprathreshold membrane potential in the simulated neuron. $C$, Afterhyperpolarization in a real cortical pyramidal cell. Following a short current injection, which evokes strong spiking activity, the neuron shows a slowly decaying afterhyperpolarization similar to that in the simulated neuron. 


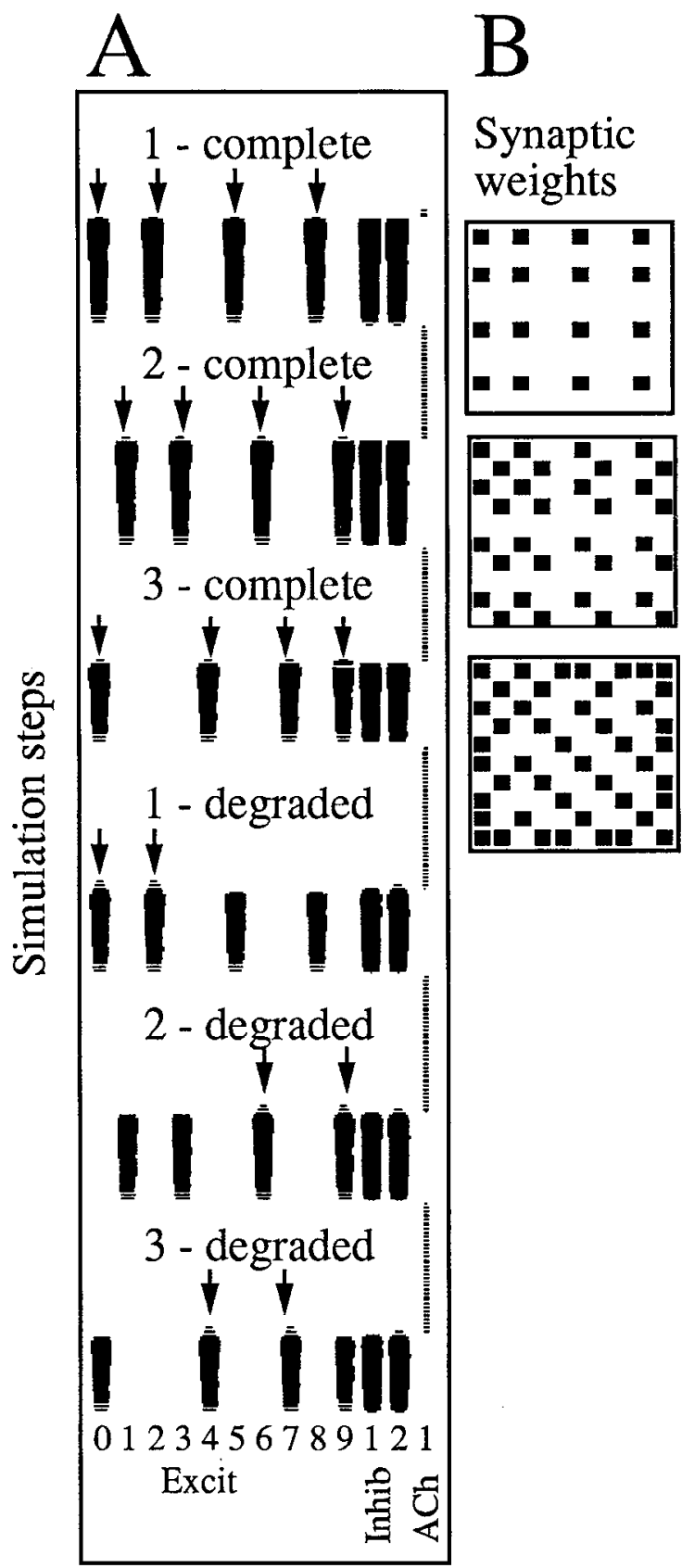

Figure 7. Sequential learning and recall of afferent input patterns in the auto-associative network, with termination of activity due to adaptation. The activity of 10 excitatory neurons, 2 inhibitory neurons, and 1 cholinergic neuron is shown during sequential presentation of complete and degraded afferent input patterns. Width of the black line represents level of activity in each neuron. Arrows indicate which neurons are receiving afferent input for each pattern (for $100 \mathrm{msec}$ ). Subsequent activity is self-sustaining until adaptation becomes sufficiently strong to knock the network out of its current activity state. On the left, the synaptic connectivity within the network is shown after presentation of each new afferent input pattern.

pression of synaptic transmission at the synapses of the longitudinal association fibers terminating in stratum radiatum of CA3. Physiological recording demonstrates that the cholinergic agonist carbachol strongly suppresses extracellularly recorded synaptic potentials in stratum radiatum of hippocampal region CA3. As shown in Figure 8, $100 \mu \mathrm{M}$ carbachol results in reduc- tion of the height and rising slope of stratum radiatum synaptic potentials by about $80 \%$.

The dose-response curve for suppression of synaptic potentials in region CA3 is shown in Figure 9. The average suppression of the rising slope of synaptic potentials was $16.8 \pm 9.8 \%$ at $1 \mu \mathrm{M}$ (percent change \pm standard error) $(n=5), 40.5 \pm$ $7.7 \%$ at $5 \mu \mathrm{M}(n=5), 65.8 \pm 3.7 \%$ at $20 \mu \mathrm{M}(n=5)$, and $76.7 \pm 4.3 \%$ at $100 \mu \mathrm{M}(n=12)$. The dose-response curve for stratum radiatum in region $\mathrm{CA} 3$ resembles that obtained in stratum radiatum of hippocampal region $\mathrm{CA}$. Data from paired pulse stimulation was gathered for measurement of the change in paired-pulse facilitation during suppression of synaptic transmission. During suppression of synaptic transmission by $100 \mu \mathrm{M}$ carbachol, the percent paired-pulse facilitation increased by $31.4 \%$. This increase in paired-pulse facilitation has been taken to suggest that suppression is mediated by presynaptic receptors (see Hasselmo and Bower, 1992).

\section{Discussion}

In the network presented here, the interaction of feedback excitation and feedback inhibition allows the network to show bounded, stable activity patterns that could represent memory states. This network allows analysis of the function of the cholinergic suppression of synaptic transmission at excitatory feedback synapses, as demonstrated in the experimental data from hippocampal region CA3 presented here. The level of cholinergic suppression of feedback excitation determines the extent to which the network learns components of new afferent input patterns. With high levels of cholinergic modulation, the network learns the new afferent pattern with no element of previous overlapping patterns. With low levels of cholinergic modulation, the network recalls previously stored patterns that overlap with the afferent input, without incorporating any new elements. With levels of cholinergic modulation in an intermediate range in which the feedback regulation of cholinergic modulation becomes influential, the network stores a combination of the elements of the new and old patterns.

The network shows the basic property of auto-associative memory function, responding to degraded versions of previously stored patterns with a stable attractor state matching the originally stored pattern (once afferent input is removed). Auto-associative memory function has been proposed for hippocampal region CA3 by a varicty of rescarchcrs (Marr, 1971; McNaughton and Morris, 1987; Eichenbaum and Buckingham, 1991; Treves and Rolls, 1991), but few models have been presented to demonstrate how recurrent excitation in this region could mediate recall without resulting in runaway excitatory activity. $\mathrm{Re}$ current excitatory synapses in region CA3 have also been proposed to store sequences of activity states (Marr, 1971; Levy, 1989; Minai and Levy, 1994; Prepscius and Levy, 1994). However, simulations of this activity must address the same difficulty of controlling network activity with feedback inhibition (Minai and Levy, 1994), and controlling the spread of activation across previously modified synapses during the storage of new sequences.

Similar to this model, a previously published model of the connections from region CA3 to region CA1 (the Schaffer collaterals) also demonstrates the importance of suppressing excitatory transmission at modifiable synapses during learning of new associations (Hasselmo and Schnell, 1994). In that previous article, modeling showed that heteroassociative memory function was most effective with strong cholinergic suppression at Schaf- 


\section{Control}

\section{A}
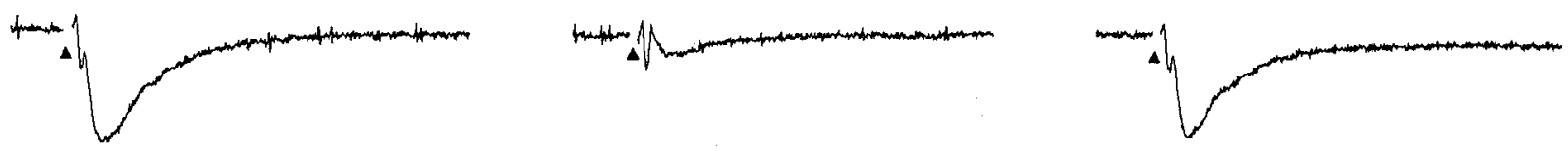

B
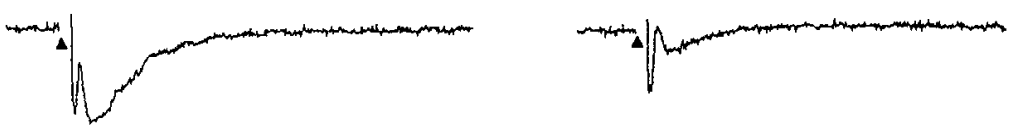

Wash

$100 \mu \mathrm{M}$ Carbachol 


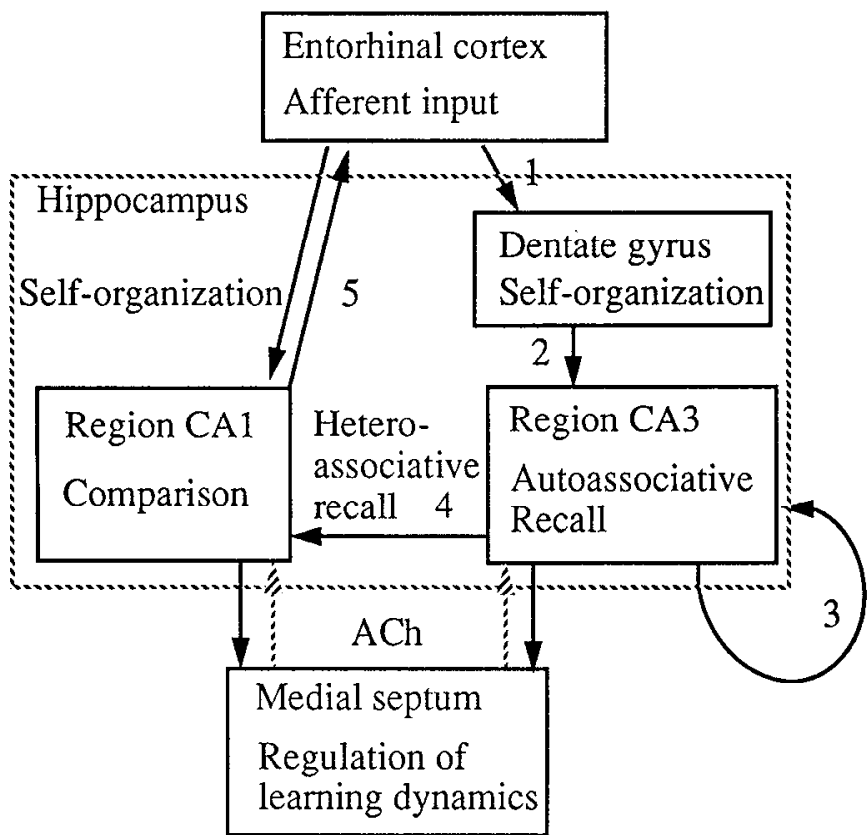

Figure 10. Schematic representation of a model of the hippocampus, showing the proposed function of individual anatomical subregions. Synaptic connections within this model mediate the following functions: $l$, Perforant path synapses in the dentate gyrus undergo self- organization to form new representations of input from entorhinal cortex. 2, Mossy fibers from dentate gyrus to $\mathrm{CA} 3$ induce a sparse pattern of activity for autoassociative storage. 3, Excitatory recurrent connections in stratum radiatum mediate auto-associative storage and recall of these patterns. 4, Schaffer collaterals from region $\mathrm{CA} 3$ to $\mathrm{CA} 1$ mediate heteroassociative storage and recall of associations between activity patterns in CA3 and the activity patterns induced by entorhinal input to region CA1. 5, Perforant path inputs to region CA1 undergo self- organization, forming new representations of entorhinal cortex input for comparison with recall from CA3. The comparison of recall activity in region $\mathrm{CA} 3$ with direct input to region $\mathrm{CA} 1$ regulates cholinergic modulation, allowing a mismatch between recall and input to increase $\mathrm{ACh}$, and a match between recall and input to decrease $\mathrm{ACh}$

stored patterns. The recall activity mediated by region $\mathrm{CA} 3$ reaches region CA1 via the Schaffer collaterals, where the levels of activity correspond to a matching of CA3 recall with the direct input from entorhinal cortex to region CA1. This comparison function regulates the level of cholinergic modulation in the network. If recall does not match direct input, cholinergic modulation is increased, setting appropriate dynamics for learning novel information. If recall matches direct input, cholinergic modulation is decreased, allowing activity to be dominated by recall. In this manner, the cholinergic levels in region CA 3 could be externally regulated, depending on how well recall matches the properties of sensory input.

Excitatory intrinsic synapses have also been proposed to mediate associative memory function in the piriform (olfactory) cortex (Hasselmo et al., 1992; Hasselmo, 1993; Hasselmo and Bower, 1993). Similar to region CA3, the piriform cortex contains excitatory recurrent synapses, but these appear to be less prevalent than in region CA3. Thus, the piriform cortex appears to have potential for both autoassociative memory function due to recurrent synapses, and heteroassociative memory function due to modifiable excitatory synapses between different subregions (for example, rostral and caudal piriform cortex). Similar to regions CA1 and CA3, the piriform cortex also demonstrates a laminar selectivity of the cholinergic suppression of synaptic transmission, with strong suppression at intrinsic and association fibers in layer Ib and an absence of suppression at afferent fibers in layer Ia (Hasselmo and Bower, 1992). The piriform cortex differs physiologically from hippocampal subregions in that it shows weaker feedback inhibition and does not contain bursting pyramidal cells. The bursting and stronger inhibition in the hippocampus may be required to maintain stability in the presence of stronger excitatory feedback.

The hippocampus and piriform cortex both play a role in the processing of olfactory information. Lesions of the fornix impair the lcarning of odor discriminations in which two odors are presented simultaneously, but not when single odors are presented sequentially (Eichenbaum et al., 1988), suggesting that this lesion impairs the encoding of relations between odor stimuli ( $\mathrm{Ei}$ chenbaum et al., 1991). Fornix lesions destroy most of the cholinergic innervation of the hippocampus, without damaging connections between the hippocampus and the entorhinal cortex. This indicates that the encoding of relations may specifically depend upon cholinergic innervation. The work presented here suggests that without cholinergic suppression of recall, it may be difficult to form distinct new representations of separate behavioral parameters or events during training. This effect would be particularly striking when many of the dimensions of the sensory stimulation appear in each training cycle-for example, in a task requiring the learning of a response to simultaneously presented odors rather than sequentially presented odors (Eichenbaum et al., 1988).

Though their position relative to the olfactory epithelium suggests a sequential processing of olfactory information, single unit recordings from the hippocampus and the piriform cortex during performance of an olfactory discrimination task suggests more interactive processing. Neurons in the piriform cortex do not only respond during sampling of olfactory cues, but also respond to other factors such as the initial approach to odor ports and to reward (Schoenbaum and Eichenbaum, 1994). Apart from a greater specificity for individual odors, these response characteristics resemble those in the hippocampus (Eichenbaum et al., 1989). This suggests a continuous dynamical interaction between the hippocampus and piriform cortex, in which individual attractors associated with components of behavior may involve excitatory feedback between simultaneously active neurons in both regions. Rather than processing information and passing it on to the hippocampus, the piriform cortex may provide additional components of a broadly distributed attractor, thereby enhancing the ability to separately represent a wide range of complex odor stimuli.

\section{Bounded attractor dynamics during recall in associative memory models}

The model presented here differs from most previous models in its ability to approach stable attractor states without use of sigmoid input-output functions, which limit activity at a maximal firing rate. These stable attractor states allow activity induced by input patterns resembling the stored pattern to result in a consistent final response. Even if neuronal adaptation or inhibition mediated by $\mathrm{GABA}_{\mathrm{B}}$ receptors causes these attractor states to decay, they still provide a mechanism for storage and recall of memory states that do not differ even with high variance in the afferent input.

Recurrent excitation can easily lead to exponential increases of excitatory activity within a region, as shown in Figure 3 . This has been prevented using a variety of techniques in various mod- 
els. In simple linear associative memories, recall consisted of only one step of activation, equivalent to matrix multiplication (Kohonen et al., 1977; Anderson, 1983; Kohonen, 1984). In later attractor neural networks, the maximal firing rate of neurons was limited by use of sigmoid input-output functions, such that during recall neurons would fire near their maximal firing rate (Hopfield, 1984; Amit, 1988). More recent efforts have focused on methods of attaining recall attractor states with low firing rates. This has been attained using threshold linear neurons by including normalization of total network activity (Treves, 1990, 1991).

Here, teedback inhibition provides a mechanism for preventing exponential increases in excitatory activity in the network. A simplified representation of shunting inhibition has previously been used to attain stable attractor states at low firing rates (Abbott, 1991, 1992). The use of shunting inhibition may provide even greater stability, and applies directly to the electrotonic structure of cortical pyramidal cells, but the framework presented here may be sufficiently realistic and has simpler threshold linear characteristics. The previous simulations of shunting inhibition did not address problems of learning, though it was noted that familiar patterns caused greater levels of activity (Abbott, 1992). The interaction of excitation and inhibition plays a role in a wide range of other abstract cortical models (Grossberg, 1970, 1972; Wilson and Cowan, 1972).

\section{Issues of physiological realism}

The model presented here uses a continuous representation of firing rate. The validity of this analysis for the function of a nctwork of spiking neurons depends upon the firing rates of neurons in attractor states being essentially asynchronous (Treves, 1993; Van Vreeswijk and Abbott, 1993) and the population of neurons being very large, particularly as the time constants of synaptic transmission become progressively smaller. Given these limitations, however, this simplified representation has advantages over networks using sigmoid input-output functions, including other attractor neural networks and networks trained with backpropagation of error, in that it does not artificially constrain the activity of neurons. This allows analysis of how various physiologically realistic features such as adaptation and feedback inhibition influence the firing rate of neurons, and how these influences affect the stable states of the network. Most existing models of attractor neural networks do not have a direct one to one correspondence with features of cortical networks. In addition, as mentioned above, the network presented here explicitly deals with the issue of learning, which has becn largely ignored in attractor neural networks, and which relies upon a range of unphysiological processes in networks using backpropagation of error.

The computational model presented here provides a solid theoretical framework for guiding development of simulations using integrate and fire representations, or detailed biophysical representations of cortical networks (Barkai et al., 1994; Barkai and Hasselmo, 1994; Hasselmo et al., 1994b; Hasselmo, 1995). The firing rate representation used here has greater potential for addressing the dynamics of cortical function. This framework can be used to guide modeling of the sustained neuronal firing observed with single unit recording in awake, behaving monkeys performing delayed match-to-sample tasks (Fuster, 1973; Fuster and Jervey, 1982). The dynamics of self-sustained activity presented here provide a clearer relationship to actual cortical structure than recurrent networks trained with backpropagation through time (Zipser et al., 1993). This model will also allow analysis of the network dynamics underlying the decrease in neuronal firing associated with repeated presentation of a novel visual stimulus (Rolls et al., 1989). In the simulations presented here, repeated presentation of a stimulus results in decreased response due to the currents underlying adaptation. Clearly, the actual physiological mechanisms for storage of memory states in cortical structures will prove to be considerably more complex. However, the model presented here provides a mathematical framework for more detailed biophysical models exploring the mechanisms underlying this function.

\section{Appendix}

For a homogeneous population of excitatory and inhibitory neurons, the system of differential equations shown in Equation 1 can be represented as a single excitatory neuron with excitatory synaptic feedback and inhibitory synaptic input, and a single inhibitory neuron with inhibitory synaptic reedback and excilatory synaptic input, both of which receive direct afferent input, as summarized in Figure $1 B$. This representation takes the following form:

$$
\left[\begin{array}{l}
d a / d t \\
d h / d t
\end{array}\right]=\left[\begin{array}{cc}
W-\eta & -H \\
W^{\prime} & -H^{\prime}-\eta^{\prime}
\end{array}\right]\left[\begin{array}{l}
a \\
h
\end{array}\right]+\left[\begin{array}{c}
A-W \theta_{a}+H \theta_{h} \\
A-W^{\prime} \theta_{a}+H^{\prime} \theta_{h}
\end{array}\right]
$$

Note that this is only valid for values of $a$ above $\theta_{a}$ and values of $h$ above $\theta_{h}$. When membrane potentials fall below threshold, the synaptic weights arising from the neuron should be set to zero to represent the solution in that region.

The eigenvalues of this system of equations take the form

$$
\lambda_{1}, \lambda_{2}=\frac{T \pm \sqrt{T^{2}-4 K}}{2}
$$

where the trace $T=(W-\eta)+\left(-H^{\prime}-\eta^{\prime}\right)$, the determinant $K=(W-\eta)\left(-H^{\prime}-\eta^{\prime}\right)+W^{\prime} H$ and the discriminant $R=T^{2}$ $-4 K=\left((W-\eta)-\left(-H^{\prime}-\eta\right)\right)^{2}-4 W^{\prime} H$.

For stable self-sustained activity states to exist in the region above threshold, the trace must be less than zero $(T<0)$, and the determinant must be greater than zero $(K>0)$. This provides the stability criteria presented in the results and in Figure 3. For values of the discriminant $R$ less than zero, the solution shows damped oscillations toward equilibrium, while values of $R$ greater than zero give strong damping preventing any oscillations (reviewed in pp. 13-16 of Jordan and Smith, 1987; or pp. 3336 of Hirsch and Smale, 1974).

The associated eigenvectors are

$$
\left[\begin{array}{l}
x_{1} \\
y_{1}
\end{array}\right]=\left[\begin{array}{c}
1 \\
\left(T^{+}-\sqrt{R}\right) / 2 H
\end{array}\right] \text { and }\left[\begin{array}{l}
x_{2} \\
y_{2}
\end{array}\right]=\left[\begin{array}{c}
1 \\
\left(T^{+}+\sqrt{R}\right) / 2 H
\end{array}\right] \text {, }
$$

where $T^{+}=(W-\eta)+\left(-H^{\prime}-\eta^{\prime}\right)$. The equilibrium state of the network $Q$ can be found by setting $d a / d t=d h / d t=0$. The equilibrium $Q$ has the value

$$
Q=\frac{A-W \theta_{a}+H \theta_{h}+\frac{\left(H W^{\prime} \theta_{a}-H A^{\prime}-H H^{\prime} \theta_{h}\right)}{\eta^{\prime}+H^{\prime}}}{\eta-W+\frac{H W^{\prime}}{\eta^{\prime}+H^{\prime}}}
$$

Solving the nonhomogeneous system for $R>0$ and for the initial conditions $a(0)=a_{0}$, and $c(0)=c_{0}$, yields the solution 


$$
\begin{aligned}
a=Q+ & {\left[\left(a_{0}+Q\right) \frac{y_{2}}{y_{1}+y_{2}}\right.} \\
& \left.-\left(c_{0}-W^{\prime}\left(Q-\theta_{a}\right) /\left(H^{\prime}+\eta^{\prime}\right)\right) \frac{1}{y_{1}+y_{2}}\right] e^{\lambda_{1} t} \\
+ & {\left[\left(c_{0}-W^{\prime}\left(Q-\theta_{a}\right) /\left(H^{\prime}+\eta^{\prime}\right)\right) \frac{2 y_{1}+y_{2}}{\left(y_{1}+y_{2}\right) y_{2}}\right.} \\
c=\frac{\gamma_{c}}{\Omega}\left(Q-\theta_{c}\right)+\left[a_{0}-Q\right) \frac{y_{2}}{y_{1}-y_{2}} & \left.-\left(c_{0}-\gamma_{c}\left(Q-\theta_{c}\right) / \Omega\right) \frac{1}{y_{1}+y_{2}}\right] y_{1} e^{\lambda_{1} t} \\
+ & {\left[\left(c_{0}-\gamma_{c}\left(Q-\theta_{c}\right) / \Omega\right) \frac{2 y_{1}+y_{2}}{\left(y_{1}+y_{2}\right) y_{2}}-\left(a_{0}-Q\right) y_{1}\right] y_{2} e^{\lambda_{2} t}, }
\end{aligned}
$$

where the constants are as defined above.

With the addition of adaptation in the equations, the eigenvectors become very complicated. However, the equilibrium condition can be calculated by setting $d a / d t=d h / d t=d c / d t=$ 0 . This yields the following equilibrium state:

$$
a=Q=\frac{A-W \theta_{a}+H \theta_{h}+\frac{H W^{\prime} \theta_{a}-H A^{\prime}-H H^{\prime} \theta_{h}}{\eta^{\prime}+H^{\prime}}+\frac{\mu \gamma}{\Omega} \theta_{c}}{\eta-W+\frac{H W^{\prime}}{\eta^{\prime}+H^{\prime}}+\frac{\mu \gamma}{\Omega}}
$$

In most simulations, the effects of adaptation moved the equilibrium state to values below the output threshold of neurons, removing the system from a nonzero attractor state.

\section{References}

Abbott LF (1991) Realistic synaptic inputs for model neural networks. Network 2:245-258.

Abbott LF (1992) Firing-rate models for neural populations. In: Neural networks: from biology to high-energy physics (Benhar O, Bosio C, Giudice PD, Tabet E, eds). Pisa: ETS Editrice.

Amaral DG, Witter MP (1989) The 3-dimensional organization of the hippocampal formation-a review of anatomical data. Neuroscience 31:571-591.

Amit DJ (1988) Modeling brain function: the world of attractor neural networks. Cambridge: Cambridge UP.

Amit DJ, Treves A (1989) Associative memory neural networks with low temporal spiking rates. Proc Natl Acad Sci USA 86:7671-7673.

Anderson JA (1983) Cognitive and psychological computation with neural models. IEEE Trans Systems Man Cybern 13:799-815.

Ault B, Nadler JV (1982) Baclofen selectively inhibits transmission at synapses made by axons of CA3 pyramidal cells in the hippocampal slice. J Pharmacol Exp Ther 223:291-297.

Barkai E, Hasselmo ME (1994) Modulation of the input/output function of rat piriform cortex pyramidal cells. J Neurophysiol 72:644658.

Barkai E, Bergman RE, Horwitz G, Hasselmo ME (1994) Modulation of associative memory function in a biophysical simulation of rat piriform cortex. J Neurophysiol 72:659-677.

Benardo LS, Prince DA (1982) Ionic mechanisms of cholinergic excitation in mammalian hippocampal pyramidal cells. Brain Res 249: 333-344.

Burgard EC, Sarvey JM (1990) Muscarinic receptor activation facilitates the induction of long-term potentiation (LTP) in the rat dentate gyrus. Neurosci Lett 116:34-39.

Carpenter GA, Grossberg S (1993) Normal and amnesic learning, rec- ognition and memory by a neural model of cortico-hippocampal interactions. Trends Neurosci 16:131-137.

Douglas RJ, Martin KAC (1990) Neocortex. In: Synaptic organization of the brain (Shepherd GM, ed), pp 389-438. New York: Oxford UP.

Eichenbaum H, Buckingham J (1990) Studies on hippocampal processing: experiment, theory and model. In: Learning and computational neuroscience: foundations of adaptive networks (Gabriel $M$, Moore J, eds), pp 171-231. Cambridge, MA: MIT Press.

Eichenbaum H, Fagan A, Mathews P, Cohen N (1988) Hippocampal system dysfunction and odor discrimination learning in rats: impairment or Facilitation depending on representational demands. Behav Neurosci 102:331-339.

Eichenbaum H, Mathews OP, Cohen N (1989) Further studies of hippocampal representation during odor discrimination learning. Behav Neurosci 103:1207-1216.

Eichenbaum H, Otto TA, Wible CG, Piper JM (1991) Building a model of the hippocampus in olfaction and memory. In: Olfaction: a model system for computational neuroscience (Davis JL, Eichenbaum $\mathrm{H}$, eds), pp 167-210. Cambridge, MA: MIT Press.

Fuster JM (1973) Unit activity in prefrontal cortex during delayedresponse performance: neuronal correlates of transient memory. $\mathbf{J}$ Neurophysiol 36:61-78.

Fuster JM, Jervey JP (1982) Neuronal firing in the inferotemporal cortex of the monkey in a visual memory task. J Neurosci 2:361-375.

Grossberg S (19/0) Some networks that can learn, remember and reproduce any number of complicated space-time patterns, II. Stud Appl Math 49:135-166.

Grossberg S (1972) Pattern learning by functional-differential neural networks with arbitrary path weights. In: Delay and functional-differential equations and their applications (Schmitt K, ed), pp 121160. New York: Academic.

Haberly LB (1985) Neuronal circuitry in olfactory cortex: anatomy and functional implications. Chem Senses 10:219-238.

Haberly LB, Bower JM (1989) Olfactory cortex: model circuit for study of associative memory? Trends Neurosci 12:258-264.

Hasselmo ME (1993) Acetylcholine and learning in a cortical associative memory. Neural Comp 5:32-44.

Hasselmo ME (1994) Runaway synaptic modification in models of cortex: implications for Alzheimer's disease. Neural Networks 7:1340.

Hasselmo ME (1995) Neuromodulation and cortical function: modeling the physiological basis of behavior. Behav Brain Res 67:1-27.

Hasselmo ME, Bower JM (1992) Cholinergic suppression specific to intrinsic not afferent fiber synapses in rat piriform (olfactory) cortex. J Neurophysiol 67:1222-1229.

Hasselmo ME, Bower JM (1993) Acetylcholine and memory. Trends Neurosci 16:218-222.

Hasselmo ME, Schnell E (1994) Laminar selectivity of the cholinergic suppression of synaptic transmission in rat hippocampal region CA1: computational modeling and brain slice physiology. J Neurosci 14: 3898-3914.

Hasselmo ME, Rolls ET, Baylis GC (1989a) The role of expression and identity in the face-selective responses of neurons in the temporal visual cortex of the monkey. Behav Brain Res 32:203-218.

Hasselmo ME, Rolls ET, Baylis GC, Nalwa V (1989b) Object-centered encoding by face-selective neurons in the cortex in the superior temporal sulcus of the monkey. Exp Brain Res 75:417-429.

Hasselmo ME, Anderson BP, Buwer JM (1992) Cholinergic modulation of cortical associative memory function. J Neurophysiol 67: $1230-1246$.

Hasselmo ME, Schnell E, Barkai E, Berke J (1994a) A model of the hippocampus combining self-organization and associative memory function. In: Advances in neural information processing systems, vol 7.

Hasselmo ME, Barkai E, Horwitz G, Bergman RE (1994b) Modulation of neuronal adaptation and cortical associative memory function. In: Computation and neural systems (Eeckman F, Bower JM, eds). Norwell, MA: Kluwer.

Hirsch MW, Smale S (1974) Differential equations, dynamical systems, and linear algebra. New York: Academic.

Hopfield JJ (1984) Neurons with graded responses have collective computational properties like those of two-state neurons. Proc Natl Acad Sci USA 81:3088-3092.

Huerta PT, Lisman JE (1993) Heightened synaptic plasticity of hippocampal CA1 neurons during a cholinergically induced rhythmic state. Nature 364:723-725. 
Jordan DW, Smith P (1987) Nonlinear ordinary differential equations. Oxford: Clarendon.

Kelso SR, Ganong AH, Brown TH (1986) Hebbian synapses in the hippocampus. Proc Natl Acad Sci USA 83:5326-5330.

Kohonen T (1984) Self-organization and associative memory. Berlin: Springer.

Kohonen T, Lehtio P, Rovamo J, Hyvarinen I, Bry K, Vainio L (1977) A principle of neural associative memory. Neuroscience 2:10651076.

Levy WB (1989) A computational approach to hippocampal function. In: Computational models of learning in simple neural systems (Hawkins RD, Bower GH, eds), pp 243-305. Orlando, FL: Academic.

Levy WB, Colbert CM, Desmond NL (1990) Elemental adaptive processes of neurons and synapses: a statistical/computational perspective. In: Neuroscience and connectionist theory (Gluck MA, Rumelhart DE, eds), pp 187-236. Hillsdale, NJ: Erlbaum.

Madison DV, Nicoll RA (1984) Control of the repetitive discharge of rat CA1 pyramidal neurones in vitro. J Physiol (Lond) 354:319-331.

Marr D (1971) Simple memory: a theory for archicortex. Philos Trans R Soc [Biol] 262:23-81.

McClelland II, McNaughton RI, OReilly R, Nadel I. (1997) Complementary roles of hippocampus and neocortex in learning and memory. Soc Neurosci Abstr 18:508.7.

McLennan H, Miller JJ (1974a) The hippocampal control of neuronal discharges in the septum of the rat. J Physiol (Lond) 237:607-624.

McLennan H, Miller JJ (1974b) Gamma-aminobutyric acid and inhibition in the seplal nuclei of the rat. J Physiol (Lond) 237:625-633.

McNaughton BL (1991) Associative pattern completion in hippocampal circuits: new evidence and new questions. Brain Res Rev 16: $193-220$.

McNaughton BL, Morris RGM (1987) Hippocampal synaptic enhancement and information storage within a distributed memory system. Trends Neurosci 10:408-415.

Minai AA, Levy WB (1994a) Setting the activity level in sparse random networks. Neural Comp 6:83-97.

Minai AA, Levy WB (1994b) The dynamics of sparse random networks. Biol Cybern.

Otto T, Eichenbaum J (1992) Neuronal activity in the hippocampus during delayed non-match to sample performance in rats: evidence for hippocampal processing in recognition memory. Hippocampus 2:323-334

Pitler TA, Alger BE (1992) Cholinergic excitation of GABAergic interneurons in the rat hippocampal slice. J Physiol (Lond) 450:127142.

Prepscius C, Levy WB (1994) Sequence prediction and cognitive mapping by a biologically plausible neural network, pp IV-164-IV-169. San Diego: Proc World Congr Neural Nets.

Rolls ET, Baylis GC, Hasselmo ME, Nalwa V (1989) The effect of learning on the face selective responses of neurons in the cortex in the superior temporal sulcus of the monkey. Exp Brain Res 76:153164

Scanziani M, Gahwiler BH, Thompson SM (1993) Presynaptic inhi- bition of excitatory synaptic transmission mediated by $\alpha$ adrenergic receptors in area CA3 of the rat hippocampus in vitro. J Neurosci 13:5393-5401.

Schoenbaum GM, Eichenbaum H (1994) Information coding in prefrontal cortex of the behaving rodent: single unit and ensemble data in orbital prefrontal and piriform cortex. Soc Neurosci Abstr 20:808.

Tang AC, Hasselmo ME (1994) Selective suppression of intrinsic but not afferent fiber synaptic transmission by baclofen in the piriform (olfactory) cortex. Brain Res 659:75-81.

Swanson LW, Cowan WM (1979) Connections of the septal region in the rat. J Comp Neurol 186:621-656.

Toth K, Borhegyi Z, Freund TF (1993) Postsynaptic targets of GABAergic hippocampal neurons in the medial septum diagonial band of Broca complex. J Neurosci 13:3712-3724.

Traub R, Miles R, Buzsaki G (1992) Computer simulation of carbachol-driven rhythmic population oscillations in the CA3 region of the in vitro rat hippocampus. J Physiol (Lond) 451:653-672.

Treves A (1990) Threshold-linear formal neurons in auto-associative nets. J Phys [A, Math Gen] 23:2418.

Treves A (1991) Are spin-glass effects relevant to understanding realistic autoassociative memories? I Phys [A, Math Gen] 24:26452654.

Treves A (1993) Mean-field analysis of neuronal spike dynamics. Network Comput Neural Syst 4:259-284.

Treves A, Rolls ET (1991) What determines the capacity of autoassociative memories in the brain? Network 2:371-397.

Valentino RJ, Dingledine R (1981) Presynaptic inhibitory effect of acetylcholine in the hippocampus. J Neurosci 1:784-792.

Van Vreeswijk C, Abbott LF (1993) Self-sustained firing in populations of integrate-and-fire neurons. SIAM J Appl Math 53:253-264.

Vanier MC, Bower JM (1992) Noradrenergic suppression of synaptic transmission in rat piriform (olfactory) cortex. Soc Neurosci Abstr 18:1353.

Wickelgren WA (1979) Chunking and consolidation: a theoretical synthesis of semantic networks, configuring in conditioning, S-R versus cognitive learning, normal forgetting, the amnesic syndrome and the hippocampal arousal system. Psychol Rev 86:44-60.

Wiener SI, Paul CA, Eichenbaum H (1989) Spatial and behavioral correlates of hippocampal neuronal activity. J Neurosci 9:2737-2763.

Wigstrom H, Gustafsson B, Huang Y-Y, Abraham WC (1986) Hippocampal long-term potentiation is induced by pairing single afferent volleys with intracellularly injected depolarizing current pulses. Acta Physiol Scand 126:317-319.

Williams SH, Johnston D (1990) Muscarinic depression of synaptic transmission at the hippocampal mossy fiber synapse. J Neurophysiol 64:1089-1097.

Wilson HR, Cowan JD (1972) Excitatory and inhibitory interactions in localized populations of model neurons. Biophys $\mathbf{J}$ 12:1-24.

Wilson MA, McNaughton RI. (1993) Dynamics of the hippocampal ensemble code for space. Science 261:1055-1058.

Zipser D, Kehoe B, Littlewort G, Fuster J (1993) A spiking network model of short-term active memory. J Neurosci 13:3406-3420. 